\title{
Beam Patterns of the Five-hundred-metre Aperture Spherical Telescope: Optimisation
}

\author{
B. Dong ${ }^{1,2,3}$ and J. L. Han ${ }^{1}$ \\ ${ }^{1}$ National Astronomical Observatories, CAS, Jia-20 DaTun Road, Chaoyang District, Beijing 100012, China \\ ${ }^{2}$ School of Physics, University of Chinese Academy of Sciences, Beijing 100049, China \\ ${ }^{3}$ Email: dongbin@nao.cas.cn
}

(Received April 10, 2013; AcCepted June 9, 2013; Online Publication July 25, 2013)

\begin{abstract}
The Five-hundred-metre Aperture Spherical Telescope (FAST) uses adaptive spherical panels to achieve a huge collecting area for radio waves. In this paper, we try to explore the optimal parameters for the curvature radius of spherical panels and the focal distance by comparison of the calculated beam patterns. We show that to get the best beam shape and maximum gain, the optimal curvature radius of panels is around $300 \mathrm{~m}$, and a small shift in the focal distance of a few $\mathrm{cm}$ is needed. The aperture efficiency can be improved by $\sim 10 \%$ at $3 \mathrm{GHz}$ by this small shift. We also try to optimise the panel positioning for the best beam, and find that panel shifts of a few $\mathrm{mm}$ can improve the beam pattern by a similar extent. Our results indicate that accurate control of the feed and panel positions to the $\mathrm{mm}$ level is very crucial for the stability of FAST's observational performance.
\end{abstract}

Keywords: techniques: miscellaneous - telescopes

\section{INTRODUCTION}

The Five-hundred-metre Aperture Spherical Telescope (FAST) is being constructed in a karst depression in Guizhou Province as one of the mega science facilities for basic research in China (Nan et al. 2011). The spherical panels with an overall diameter of $500 \mathrm{~m}$ will be used to collect radio waves from the universe. It has about 4400 active triangular panels with a spherical surface and a curvature radius of $318.5 \mathrm{~m}$, while sitting on a cable-net that has a spherical shape and a radius of $300 \mathrm{~m}$ (Figure 1). During observations, the illuminated panels of the main reflector will be adjusted instantaneously to form a paraboloid with an aperture of $300 \mathrm{~m}$ in diameter (Qiu 1998) operating under the real-time control. The feed cabin is suspended about $140 \mathrm{~m}$ above the panels, with a focal ratio of $f I D=0.4665$. The FAST will be able to observe radio sources up to a zenith angle of $z=$ $40^{\circ}$ in a frequency range of $70 \mathrm{MHz}$ to $3 \mathrm{GHz}$. Dong \& Han (2013) recently calculated the beam patterns of the FAST at $200 \mathrm{MHz}, 1.4 \mathrm{GHz}$ and $3.0 \mathrm{GHz}$ for observations at zenith angles of $z=0^{\circ}, 27^{\circ}$ (S1 in Figure 1) and $40^{\circ}$ (S2 in Figure 1).

When spherical panels with the same curvature radius are used to fit a paraboloid, the deviation of panel surface from an ideal parabolic shape is unavoidable. Each spherical panel in the FAST leads to some axial defocusing effect, which affects the focal point and telescope gain. The curvature radius of spherical panels and the focal distance are therefore important parameters to optimise. We noticed that the early design of the spherical curvature radius is $300 \mathrm{~m}$ (Qiu 1998), and later it was officially designed to be $318.5 \mathrm{~m}$ (Nan et al. 2011) based on calculations of the minimum rootmean-square ( $r m s$ ) deviation (Nan 2006; Gan \& Jin 2010). However, the illumination function of a practical feed, gaps between panels, etc, were not taken into account, and the aperture efficiency was therefore overestimated to be as surprisingly high as $93.3 \%$ (Gan \& Jin 2010).

During the beam pattern calculation (Dong \& Han 2013), we noticed that the beam shape and telescope gain are very sensitive to the focal distance. In that paper, we calculated the beam patterns of the FAST with official parameters in Nan et al. (2011), i.e. the curvature radius of panels $\rho_{c}=318.5 \mathrm{~m}$, and the focal distance $f=300 \mathrm{~m} \times 0.4665=139.95 \mathrm{~m}$. Using a coaxial feed with an edge taper of $T_{e}=-10.7 \mathrm{~dB}$, we found the aperture efficiency at $3 \mathrm{GHz}$ as being $\sim 57 \%$, about $20 \%$ lower than that of an ideal 300-m paraboloid.

In this paper, we explore the parameter space of the curvature radius of spherical panels and the focal distance and optimise the positions of panels for the best beam shapes and the maximum gain by comparison of the calculated beam patterns of the FAST at $3 \mathrm{GHz}$. At this frequency, the performance of the FAST is more sensitive to these parameters 


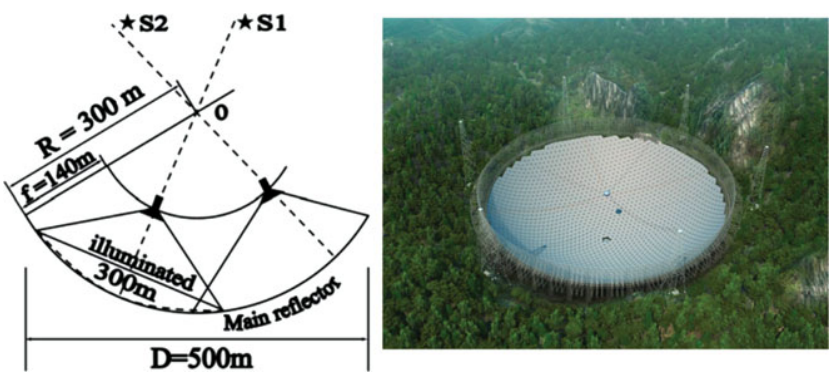

Figure 1. FAST optical geometry in the left from Nan (2006) and its 3-D model image in the right from Nan et al. (2011).

than at lower frequencies. In Section 2, we briefly describe the FAST models and feed models used in the calculations. In Section 3, we show our analyses of the focal position of the FAST and the curvature radius of a paraboloid, and present the calculation results by using the Shooting and Bouncing Ray method. Two approaches are tried to get the best beam patterns at $3 \mathrm{GHz}$. The first one is to explore the parameter space of the curvature radius of spherical panels and the focal distance, and the second is to search for the best positions of panels. Conclusions and discussions are given in Section 4.

\section{MODELS FOR FAST AND FEEDS}

The FAST is a primary-focus radio telescope. First, we describe model of the feed. For our calculation, we use the same universal coaxial horn feed as that in Dong \& Han (2013) with seven corrugated walls (Figure 2(a)), which have good symmetry in its broad radiation patterns (Figure 2(b)), very low-level sidelobes and low-level cross-polarisation within the illumination angle. We take the radiation patterns with three possible edge tapers of $T_{e}=-9.6 \mathrm{~dB}, T_{e}=-10.7 \mathrm{~dB}$ and $T_{e}=-12 \mathrm{~dB}$ by slightly changing the flare angle of the corrugated walls (Figure 2(c)).

The main reflector of the FAST consists of 4400 triangular spherical panels. The feed illuminates only some of the panels during observations. We construct the three deformed FAST models for observations at zenith angles of $z=0^{\circ}, z=27^{\circ}$ and $z=40^{\circ}$ (Figure 3), exactly the same as those in Dong \& Han (2013). The spherical panels in these models are assumed to be perfect electrical conductors with no thickness. They are adjusted to form a paraboloid with an aperture of $300 \mathrm{~m}$ in diameter (Qiu 1998) during observations. In the FAST model, we include two additional parameters as variables: the panel curvature radius $\rho_{c}$ and the focal offset $\Delta f$. In the following, we will calculate the beam patterns and telescope gains for various $\rho_{c}$ and $\Delta f$ to achieve the best performance.

\section{OPTIMISATIONS AND RESULTS}

In principle, the best focal position of the FAST can be roughly determined by geometric drawings. We demonstrate this by plotting the reflected rays in Figure 4 for a 1-D 'parabolic line' which has thirty arcs as cuts of the spher-

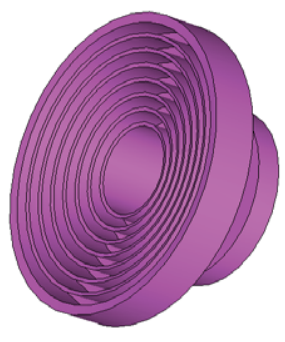

(a) 3-D model view

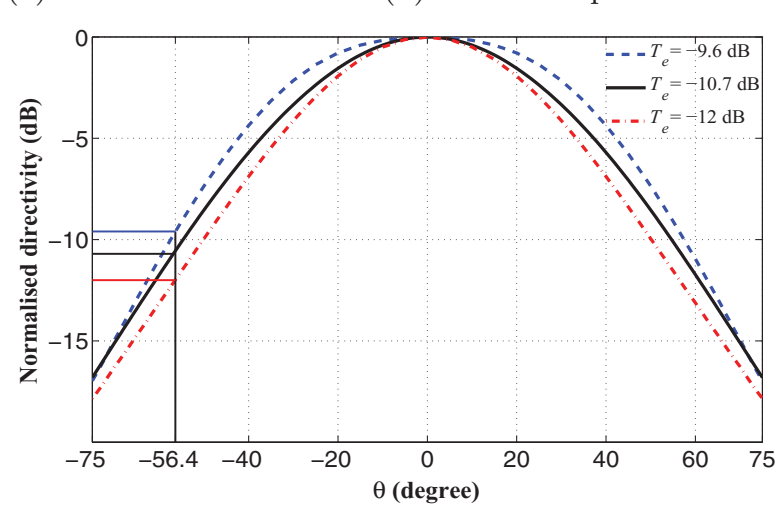

(c) Normalised patterns in the plane of $\phi=45^{\circ}$

Figure 2. Model of coaxial feed and radiation patterns with three edge tapers.

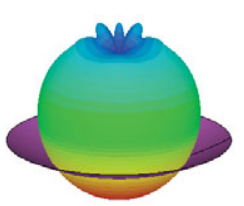

(a) ideal $300 \mathrm{~m}$ paraboloid

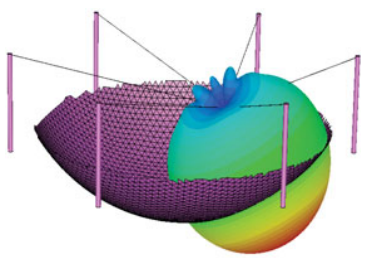

(c) $z=27^{\circ}$

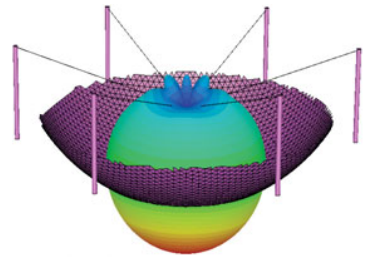

(b) $z=0^{\circ}$

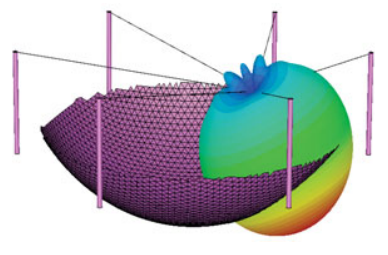

(d) $z=40^{\circ}$
Figure 3. Models for feed illumination of an ideal 300-m paraboloid and the FAST for observations at $z=0^{\circ}, z=27^{\circ}$ and $z=40^{\circ}$.

ical panels. The two ends of each arc are on a parabolic line. Ten equally spaced incident parallel rays are directed onto each arc in Figure 4(a) and the zoomed view of the focal region is shown in Figure 4(b). The best focal position should have the highest probability for the reflected rays crossing. The number of rays varies with respect to the focal distance, as shown in Figure 5. With 50000 equally spaced incident rays directed onto each arc, the peak is slightly offset from 


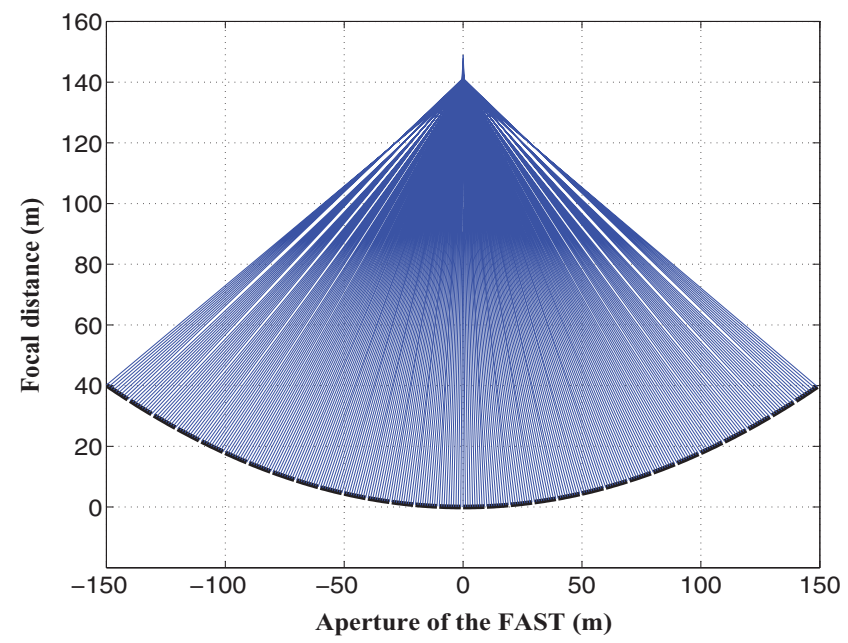

(a) Reflected rays of the 300-m paraboloid in the FAST

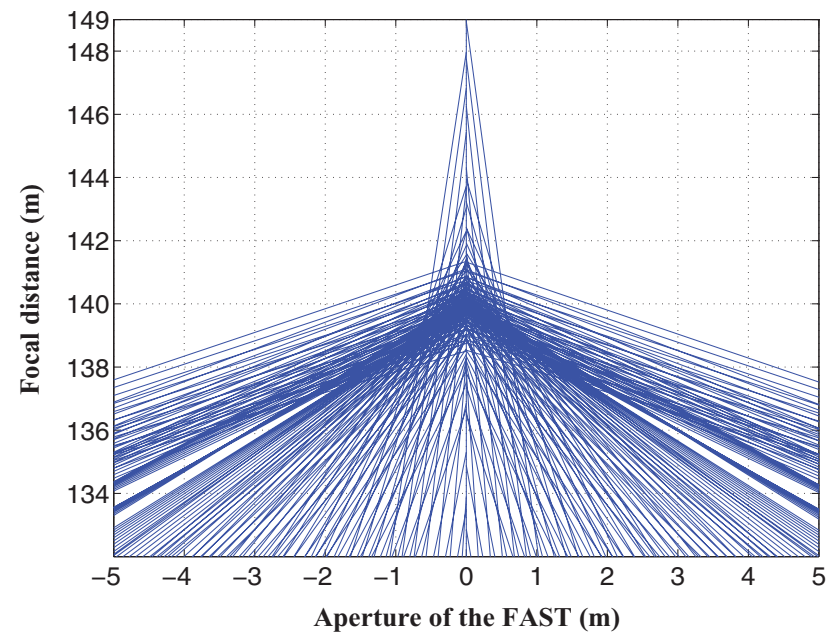

(b) zoomed view of the focal region

Figure 4. Reflected rays of an adapted paraboloid using the spherical panels for the $300 \mathrm{~m}$ aperture of the FAST.

the exact official focal distance, which is $139.95 \mathrm{~m}$ for the official focal ratio of $f / D=0.4665$.

Now, we consider the best curvature radius of the spherical panels. For a 300-m paraboloid, the curvature of a parabolic surface varies significantly with distance to the axis of the paraboloid (see Figure 6). At each point, the curvatures along the longitudinal and latitudinal lines are different. The curvature radius is about $\rho_{p}=\rho_{r}=280 \mathrm{~m}$ near the centre, while it increases to $\rho_{r}=318 \mathrm{~m}$ for the latitudinal line and $\rho_{p}=409 \mathrm{~m}$ for the longitudinal line at the aperture radius of $150 \mathrm{~m}$. Because FAST uses spherical panels of one given curvature radius of $\rho_{c}=300 \mathrm{~m}$ (Qiu 1998) or $\rho_{c}=318.5 \mathrm{~m}$ (Gan \& Jin 2010; Nan et al. 2011) to approximate a paraboloid, there must be very different deviations in the central and outer part of the mimic paraboloid due to the inconsistent curvature radii on an ideal paraboloid. Without consideration of the illumination function of a practical feed, the minimum deviation for FAST's $300 \mathrm{~m}$ aperture was found for the spherical panels having a curvature radius of $318 \mathrm{~m}$ (Gan \& Jin 2010).

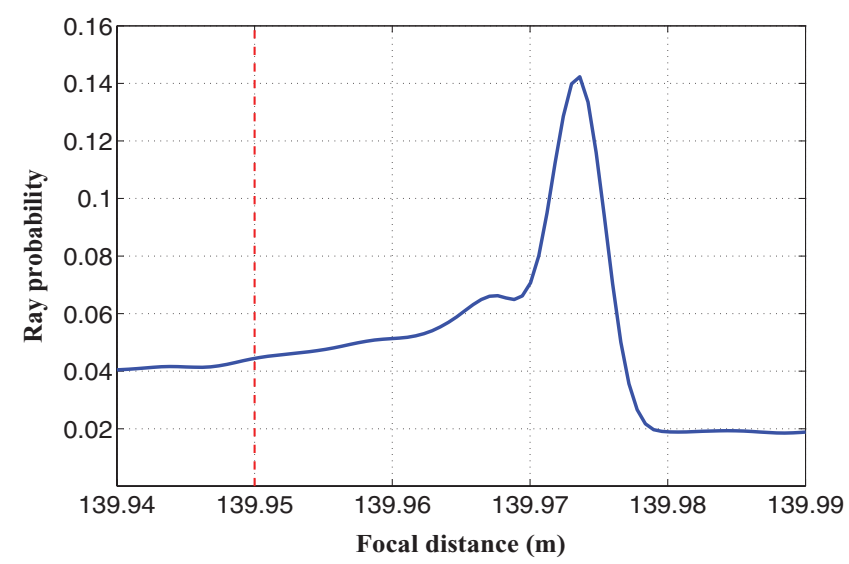

Figure 5. The probability of reflected rays passing through the region near the official focal distance of $139.95 \mathrm{~m}$.

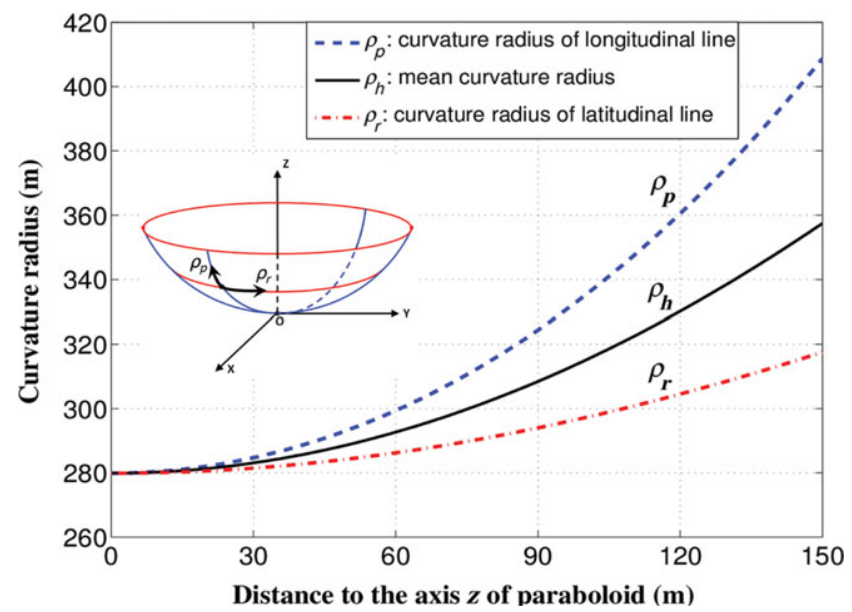

Figure 6. Variation of curvature radius of a 300-m paraboloid with $f / D=$ 0.4665 .

\subsection{Beam optimisation via panel curvature and focal offset}

We now calculate the beam patterns and the gains by using the three feed patterns and the FAST models shown in Section 2 with the Shooting and Bouncing Ray method for electromagnetic computations. We try to get the best calculation results for the parameter space of $\rho_{c}$ and $\Delta f$ in $280<\rho_{c}<350 \mathrm{~m}$ and $-1<\Delta f<9 \mathrm{~cm}$. The steps for the two parameters are $5 \mathrm{~m}$ for $\rho_{c}$ and $1 \mathrm{~cm}$ for $\Delta f$, with interpolations for some specific known values if necessary. In our calculation, each spherical panel is meshed by at least 1500 curved triangular elements, and the smallest element in critical areas has an edge length of $0.4 \mathrm{~mm}$. A total number of $10^{8}$ rays are directed from the feed with a ray distance of $0.1 \sim 50 \mathrm{~mm}$ on the spherical panel after 'adaptive ray sampling'. Experiments show that the accuracy of these settings is high enough to detect small deviations from the spherical panels and the focal shift in the FAST. Moreover, reflections from the feed cabin are ignored in our calculation since it might affect the optimal focal distance by reflecting rays into the backlobe of the feed. 

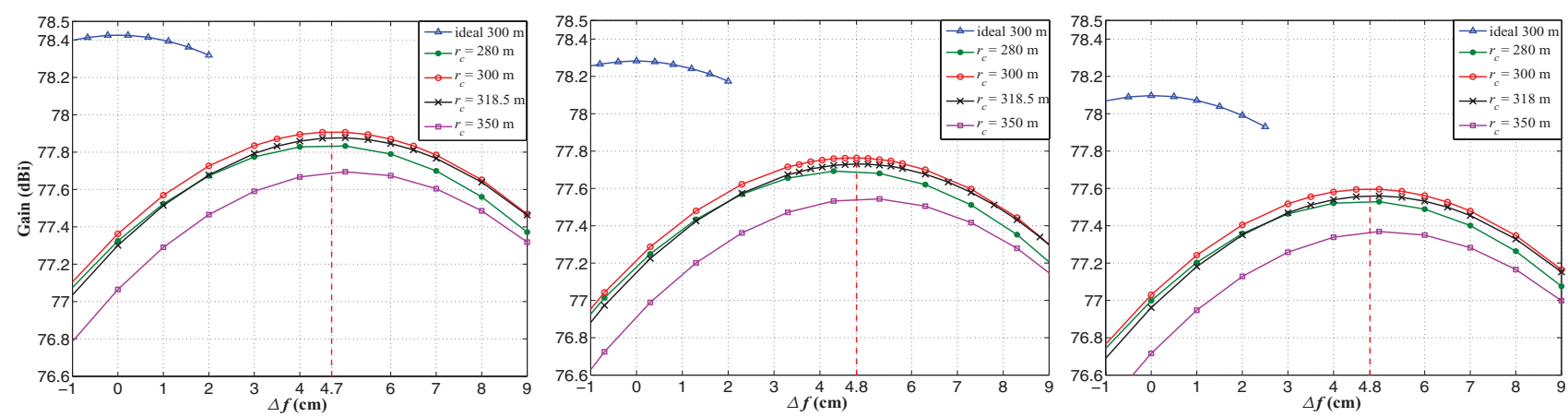

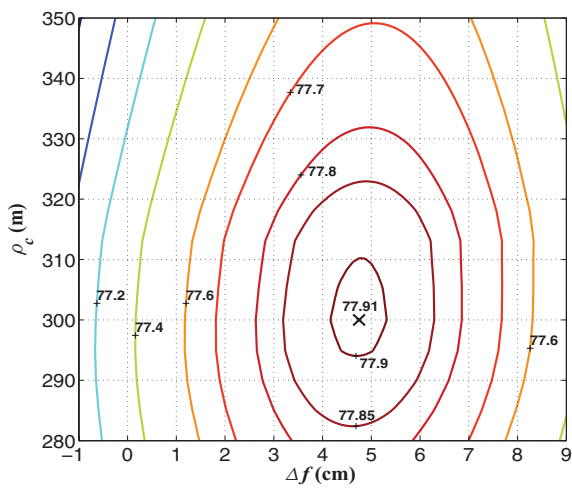

(a) $T_{e}=-9.6 \mathrm{~dB}$

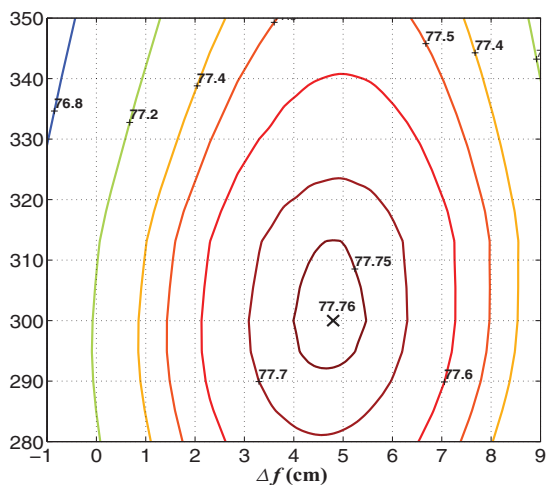

(b) $T_{e}=-10.7 \mathrm{~dB}$

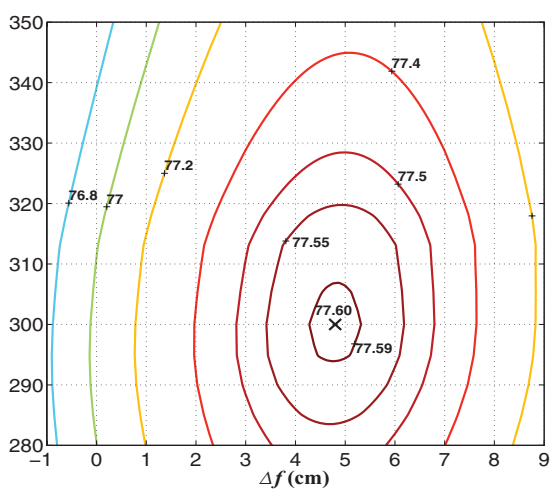

(c) $T_{e}=-12 \mathrm{~dB}$

Figure 7. Curves for the gain as a function of $\Delta f$ for $\rho_{c}=280 \mathrm{~m}, 300 \mathrm{~m}, 318.5 \mathrm{~m}$ and $350 \mathrm{~m}$ in the upper panels, and gain contours for $\Delta f$ and $\rho_{c}$ in the lower panels for three different edge tapers of the feed: $T_{e}=-9.6 \mathrm{~dB}, T_{e}=-10.7 \mathrm{~dB}$ and $T_{e}=-12.0 \mathrm{~dB}$. For comparison, the gain for the ideal $300-\mathrm{m}$ paraboloid as a function of the focal shift $\Delta f$ is also calculated and plotted as blue lines in the upper panels.

The beam patterns and telescope gains at $3 \mathrm{GHz}$ have been found to be a function of the focal shift $\Delta f$ and curvature radius $\rho_{c}$ (Figure 7). The optimal values of these two parameters are $\rho_{c}=300 \mathrm{~m}$ and $\Delta f=4.8 \mathrm{~cm}$, which are almost the same for different levels of the feed edge taper $T_{e}$. The best curvature radius, $\rho_{c}=300 \mathrm{~m}$, is smaller than $318.5 \mathrm{~m}$ obtained by Gan \& Jin (2010), because the feed illumination is considered in this paper. A lower level of feed edge taper results in smaller best gains. For the ideal $300-\mathrm{m}$ paraboloid, phase centres of the three feed patterns are carefully calculated and placed at the focal point exactly, so there are almost no focal shifts and the maximum gains are achieved at $\Delta f=$ $0 \mathrm{~cm}$. However, for FAST, a focal shift of about $4.8 \mathrm{~cm}$ is needed for the best gain. This small shift is quite critical for the performance at $3 \mathrm{GHz}$, not only showing that telescope gain could be improved by $0.6 \mathrm{~dB}$, but also indicating that the tracking of a radio source for flux measurements needs good stability in the focal distance control.

The beam patterns calculated using the official values of $\rho_{c}=318.5 \mathrm{~m}$ and $\Delta f=0 \mathrm{~cm}$ are compared with those calculated with the best values in Figure 8. The shapes of the central beam are very different from $z=0^{\circ}$ up to $z=40^{\circ}$ and get much sharper now, as listed in Table 1; the telescope gain is $77.76 \mathrm{dBi}$ at $z=0^{\circ}$, corresponding to an aperture efficiency of $67.11 \%$. Compared to the beams calculated by using the official values, the efficiency is improved by $9.6 \%$. Around the main beam at $z=0^{\circ}$, the pentagram sidelobes are caused by the pentagon-jointed panels of the main reflector of the FAST (see Figure 2 in Dong \& Han 2013).

\subsection{Beam optimisation via panel positioning}

The calculations above and in Dong \& Han (2013) are all based on the assumption that the three vertexes of each triangular panel sit on a 300-m paraboloid with $f / D=0.4665$ (see Figure 9). However, by adjusting panel positions via actuators in the 2300 control nodes, the surface deviation $(\mathrm{rms})$ of the spherical panels from the expected paraboloid can be minimised. The panel 'position optimisation' (Gan \& Jin 2010) is very necessary during the operation of the FAST.

For each panel, the surface deviation rms should be calculated from the offset $\Delta D$ in every position in the panel from the expected paraboloid. The offset $\Delta D$ should be measured perpendicular to the local surface of the paraboloid (see Figure 9). In our FAST model, considering that every position of each inclined panel has a very similar inclined angle $(\alpha)$ to the vertical $Z$-axis, we can minimise the surface deviation by searching for one offset in $Z$ directions, $\Delta Z=\Delta D / \cos \alpha$, through

$$
\begin{aligned}
\min (r m s) & =\min \left(\sqrt{\frac{\sum_{i=1}^{n} \Delta D_{i}^{2}}{n}}\right) \\
& \approx \min \left(\sqrt{\frac{\sum_{i=1}^{n}\left\{\left[\left(Z_{i}^{s}+\Delta Z\right)-Z_{i}^{p}\right] \cos \alpha\right\}^{2}}{n}}\right) .
\end{aligned}
$$



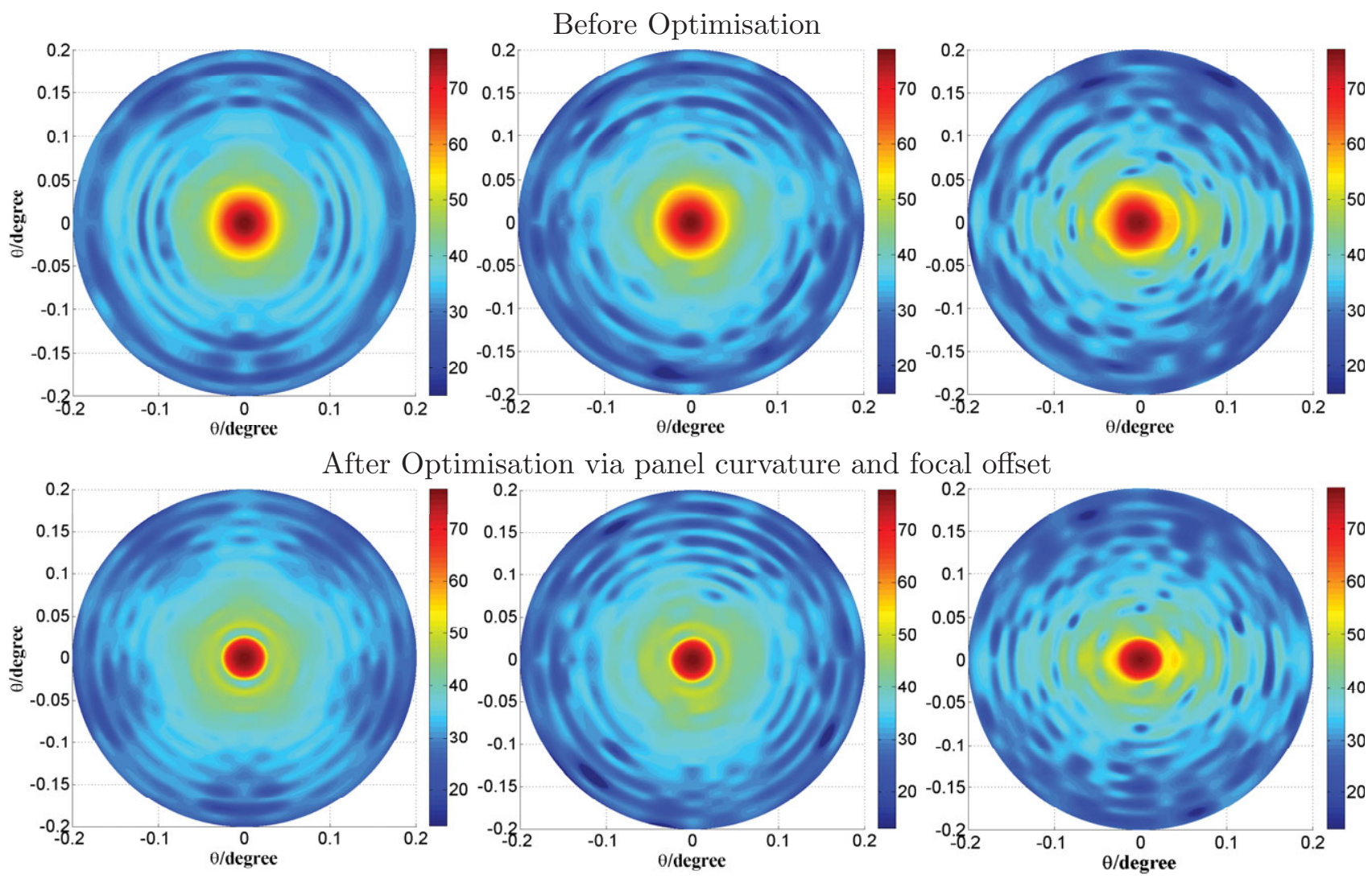

After Optimisation via panel positioning
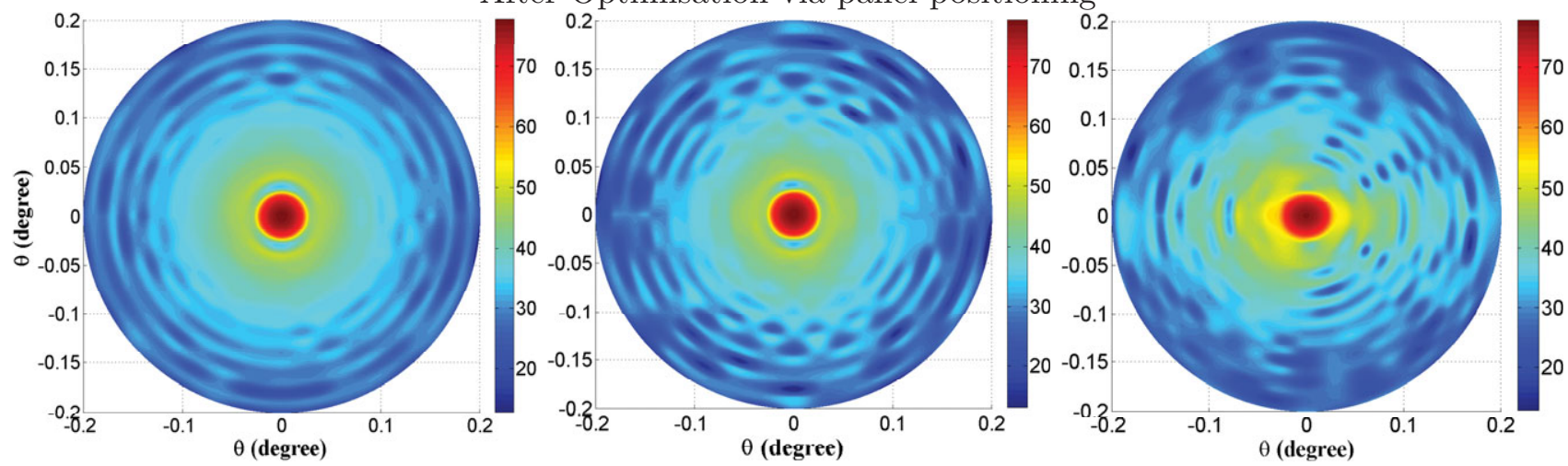

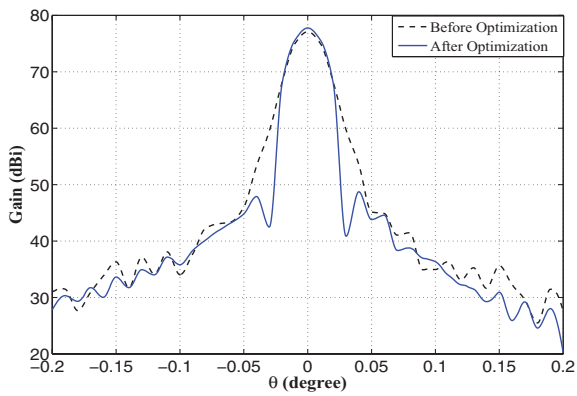

(a) $z=0^{\circ}$

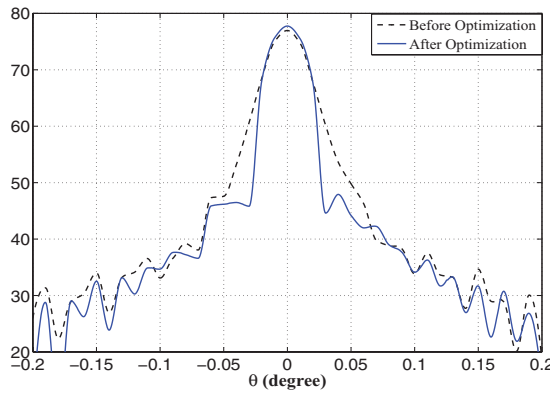

(b) $z=27^{\circ}$

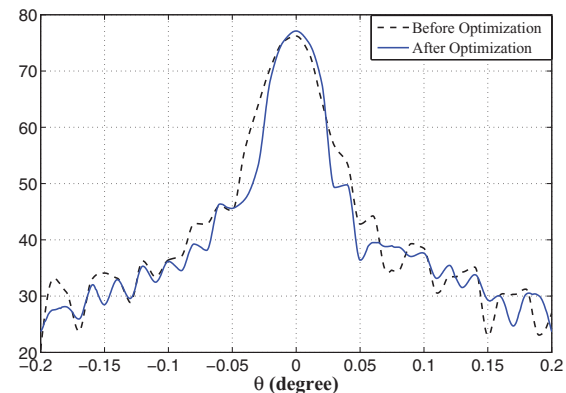

(c) $z=40^{\circ}$

Figure 8. The beam patterns of the FAST at $3 \mathrm{GHz}$ for observations at $z=0^{\circ}, 27^{\circ}$ and $40^{\circ}$ with a feed of $T_{e}=-10.7 \mathrm{~dB}$. Cuts in the $\phi=45^{\circ}$ plane are shown in the last row for beams before and after the optimisation. 
Table 1. Beam performance of the FAST at $3 \mathrm{GHz}$ before and after optimisation.

\begin{tabular}{|c|c|c|c|c|}
\hline Beam performance & 300-m paraboloid & $z=0^{\circ}$ & $z=27^{\circ}$ & $z=40^{\circ}$ \\
\hline \multicolumn{5}{|c|}{ Before optimisation } \\
\hline Gain $(G)$ & $78.28 \mathrm{dBi}$ & $77.10 \mathrm{dBi}$ & $76.96 \mathrm{dBi}$ & $76.05 \mathrm{dBi}$ \\
\hline Cross-polarisation $\left(\phi=45^{\circ}\right)$ & $-49.62 \mathrm{~dB}$ & $-49.25 \mathrm{~dB}$ & $-49.74 \mathrm{~dB}$ & $-29.80 \mathrm{~dB}$ \\
\hline First sidelobe & $-29.58 \mathrm{~dB}$ & $-34.61 \mathrm{~dB}$ & $-30.16 \mathrm{~dB}$ & $-24.76 \mathrm{~dB}$ \\
\hline HPBW & $1.41^{\prime}$ & $1.48^{\prime} \sim 1.5^{\prime}$ & $1.41^{\prime} \sim 1.44^{\prime}$ & $1.52^{\prime} \sim 1.62^{\prime}$ \\
\hline Aperture efficiency $\left(\varepsilon_{\mathrm{ap}}\right)$ & $75.65 \%$ & $57.55 \%$ & $55.82 \%$ & $59.29 \%$ \\
\hline Effective diameter $\left(d_{\text {eff }}\right)$ & $260.92 \mathrm{~m}$ & $227.59 \mathrm{~m}$ & $223.95 \mathrm{~m}$ & $202.07 \mathrm{~m}$ \\
\hline \multicolumn{5}{|c|}{ After optimisation via panel curvature and focal offset } \\
\hline $\operatorname{Gain}(G)$ & $78.28 \mathrm{dBi}$ & $77.76 \mathrm{dBi}$ & $77.75 \mathrm{dBi}$ & $77.11 \mathrm{dBi}$ \\
\hline Cross-polarisation $\left(\phi=45^{\circ}\right)$ & $-49.62 \mathrm{~dB}$ & $-51.61 \mathrm{~dB}$ & $-51.16 \mathrm{~dB}$ & $-30.55 \mathrm{~dB}$ \\
\hline First sidelobe & $-29.58 \mathrm{~dB}$ & $-28.23 \mathrm{~dB}$ & $-28.58 \mathrm{~dB}$ & $-21.36 \mathrm{~dB}$ \\
\hline HPBW & $1.41^{\prime}$ & $1.44^{\prime}$ & $1.43^{\prime}$ & $1.35^{\prime} \sim 1.62^{\prime}$ \\
\hline $\operatorname{Aperture}$ efficiency $\left(\varepsilon_{\mathrm{a} p}\right)$ & $75.65 \%$ & $67.11 \%$ & $66.91 \%$ & $67.49 \%$ \\
\hline Effective diameter $\left(d_{\text {eff }}\right)$ & $260.92 \mathrm{~m}$ & $245.77 \mathrm{~m}$ & $245.40 \mathrm{~m}$ & $228.22 \mathrm{~m}$ \\
\hline \multicolumn{5}{|c|}{ After optimisation via panel positioning } \\
\hline $\operatorname{Gain}(G)$ & $78.28 \mathrm{dBi}$ & $77.78 \mathrm{dBi}$ & $77.75 \mathrm{dBi}$ & $77.05 \mathrm{dBi}$ \\
\hline Cross-polarisation $\left(\phi=45^{\circ}\right)$ & $-49.62 \mathrm{~dB}$ & $-51.35 \mathrm{~dB}$ & $-51.03 \mathrm{~dB}$ & $-30.25 \mathrm{~dB}$ \\
\hline First sidelobe & $-29.58 \mathrm{~dB}$ & $-28.68 \mathrm{~dB}$ & $-28.65 \mathrm{~dB}$ & $-22.25 \mathrm{~dB}$ \\
\hline HPBW & $1.41^{\prime}$ & $1.44^{\prime}$ & $1.44^{\prime}$ & $1.37^{\prime} \sim 1.64^{\prime}$ \\
\hline Aperture efficiency $\left(\varepsilon_{\mathrm{ap}}\right)$ & $75.65 \%$ & $67.36 \%$ & $66.96 \%$ & $68.37 \%$ \\
\hline Effective diameter $\left(d_{\text {eff }}\right)$ & $260.92 \mathrm{~m}$ & $246.22 \mathrm{~m}$ & $245.49 \mathrm{~m}$ & $226.65 \mathrm{~m}$ \\
\hline
\end{tabular}

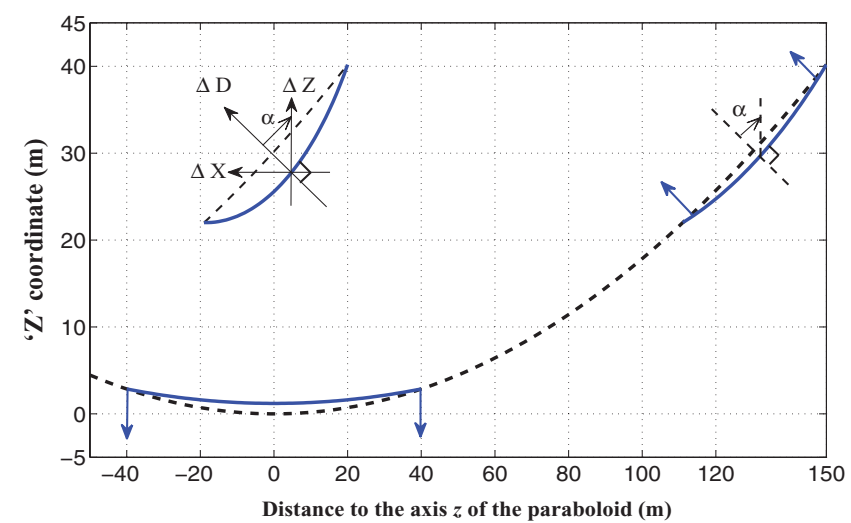

Figure 9. Illustration of panel offsets in different parts of the expected 300-m paraboloid of the FAST.

In our calculation for each panel, about $n \approx 200$ equally spaced points are sampled. $Z^{s}{ }_{i}$ and $Z^{p}{ }_{i}$ are the ' $Z$ ' coordinates of the spherical panel and expected paraboloid at the $i$ th sampling point. This makes our calculation easy, while in practice actuators have to adjust the positions of the cable-net to the expected paraboloid with a shift of $\Delta D=\Delta Z \cos \alpha$. We derived the values of $\Delta D$ which make the rms smallest for panels in various part of the expected paraboloid, as listed in Table 2. The spherical panels should be lower by several $\mathrm{mm}$ in the central region, and lift up by several $\mathrm{mm}$ at the edge (see illustration in Figure 9).

Using this new FAST model of adjusted panels, we calculate the beam patterns and telescope gains at $3 \mathrm{GHz}$ for observations at zenith angles of $z=0^{\circ}, z=27^{\circ}$ and $z=40^{\circ}$. Gain curves as a function of $\Delta f$ for $\rho_{c}=280 \mathrm{~m}, 300 \mathrm{~m}$, $318.5 \mathrm{~m}$ and $350 \mathrm{~m}$ are calculated as shown in Figure 10. These curves exhibit almost the same best performance as that in Figure 7 but the focal shift needed is very small, in the range of $\Delta f=0 \sim 1 \mathrm{~cm}$. The best curvature radius (see Figure 10) is also found to be $\sim 300 \mathrm{~m}$ for the maximum gain, very similar to that in Figure 7 . The beam pattern is also very similarly optimised as shown in Figure 8. This means that the precision in positioning of panels to achieve an accuracy of $1 \mathrm{~mm}$ is needed for the best performance for the FAST.

\section{CONCLUSIONS}

Our calculations of the beam patterns for the FAST model with a practical feed show that the best value of the curvature radius of spherical panels should be $\sim 300 \mathrm{~m}$. The feed should shift $4.8 \mathrm{~cm}$ up to get the best gain and best beam shapes. These values are slightly different from the official settings of the FAST (Nan et al. 2011; Gan \& Jin 2010), but not very significant. However, we see that the aperture efficiency could be improved by $\sim 10 \%$ at $3 \mathrm{GHz}$ and that the beam shapes become sharper with the optimised panel curvature and feed position, in addition to the gain increasing by $0.6 \mathrm{~dB}$. We also tried the best positioning of panels, and found that similar best beam patterns could be achieved through adjusting panel positions by a few mm. Our beam calculation results suggest that in future FAST tracking observations, an excellent stability and accurate positioning of feed movements to a few $\mathrm{mm}$ and of panels to $1 \mathrm{~mm}$ or better, 
Table 2. Panel offsets $\Delta D$ (unit: $\mathrm{mm})$ at different parts $(R=0 \sim 150 \mathrm{~m})$ for the best panel positioning of the mimic $300-\mathrm{m}$ paraboloid of FAST.

\begin{tabular}{lrrrrrrrr}
\hline \hline$R$ & $0 \mathrm{~m}$ & $20 \mathrm{~m}$ & $40 \mathrm{~m}$ & $60 \mathrm{~m}$ & $80 \mathrm{~m}$ & $100 \mathrm{~m}$ & $120 \mathrm{~m}$ & $150 \mathrm{~m}$ \\
\hline$\rho_{c}=280 \mathrm{~m}$ & 0 & 0.4 & 1.0 & 2.3 & 4.7 & 6.2 & 8.3 & 9.2 \\
$\rho_{c}=300 \mathrm{~m}$ & -3.6 & -3.3 & -2.3 & -1.4 & 0.9 & 2.6 & 4.8 & 5.7 \\
$\rho_{c}=310 \mathrm{~m}$ & -5.2 & -4.9 & -4.0 & -3.0 & -0.9 & 0.9 & 3.1 & 4.2 \\
$\rho_{c}=318.5 \mathrm{~m}$ & -6.6 & -6.3 & -5.4 & -4.4 & -2.3 & -0.4 & 1.7 & 2.9 \\
$\rho_{c}=350 \mathrm{~m}$ & -10.9 & -10.6 & -9.7 & -8.7 & -6.9 & -4.6 & -2.6 & -1.3 \\
\hline \hline
\end{tabular}
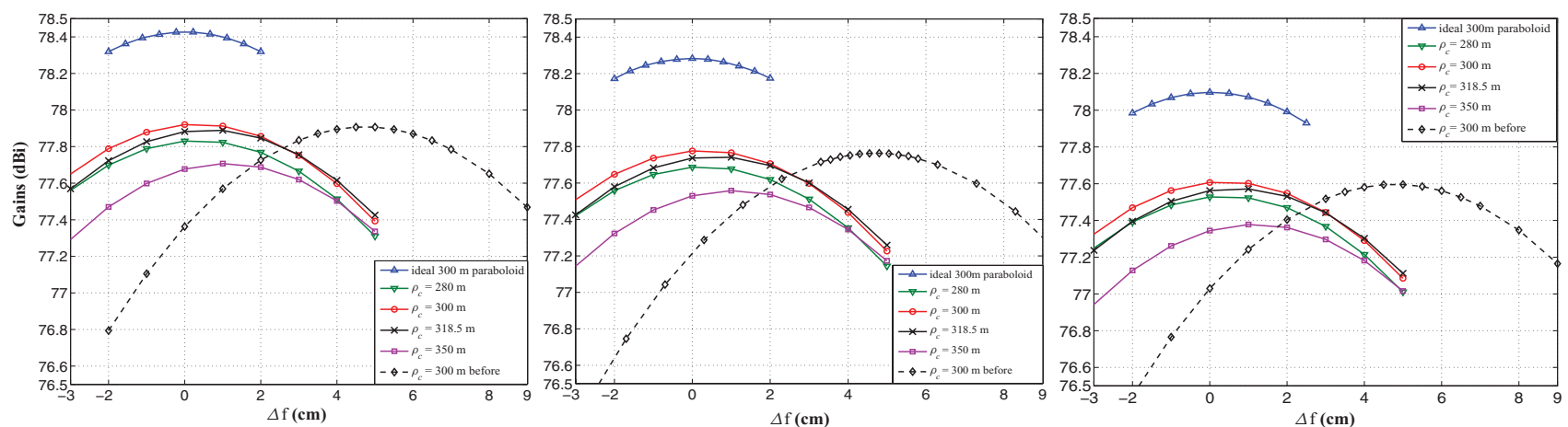

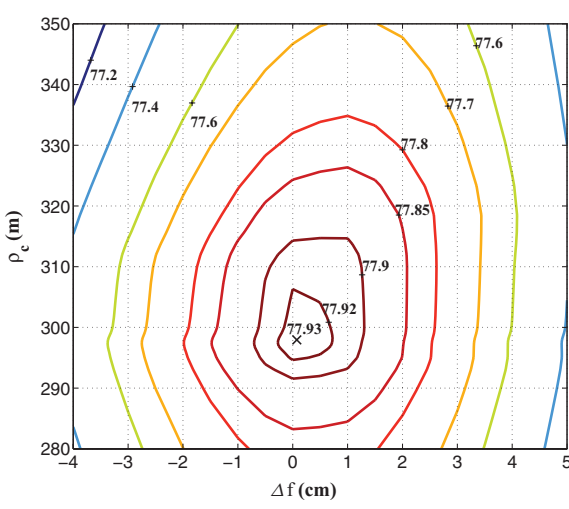

(a) $T_{e}=-9.6 \mathrm{~dB}$

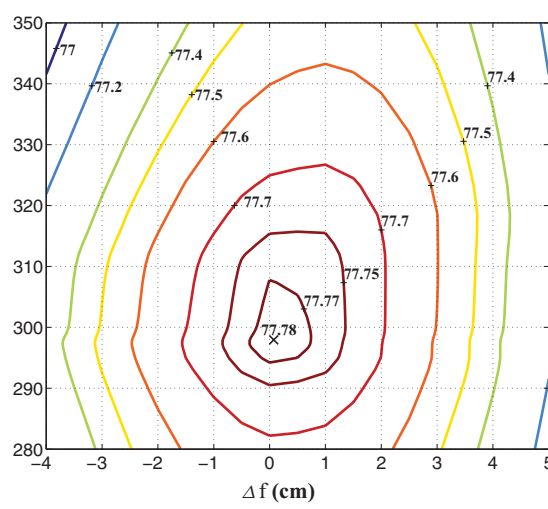

(b) $T_{e}=-10.7 \mathrm{~dB}$

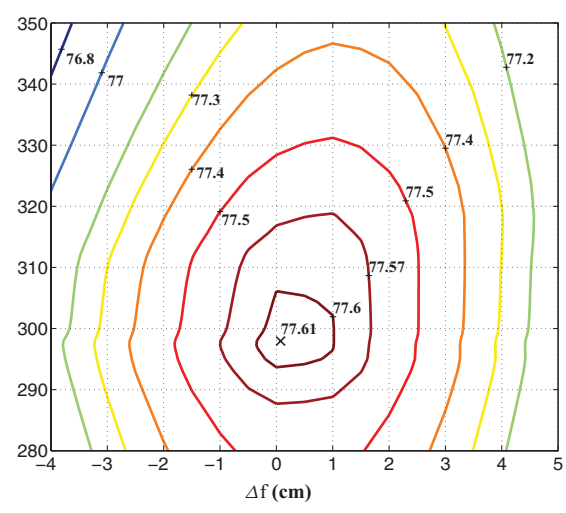

(c) $T_{e}=-12 \mathrm{~dB}$

Figure 10. After the best positioning of panels, gain curves are calculated as a function of $\Delta f$ for $\rho_{c}=280 \mathrm{~m}, 300 \mathrm{~m}, 318.5 \mathrm{~m}$ and $350 \mathrm{~m}$ in the upper panels, and gain contours for $\Delta f$ and $\rho_{c}$ in the lower panels are calculated for three different edge tapers of the feed: $T_{e}=-9.6 \mathrm{~dB}, T_{e}=-10.7 \mathrm{~dB}$ and $T_{e}=-12.0 \mathrm{~dB}$. For comparison, the gain for the ideal $300-\mathrm{m}$ paraboloid as a function of the focal shift $\Delta f$ is calculated and plotted as blue lines in the upper panels.

have to be provided so that the observational beams do not obviously vary.

\section{ACKNOWLEDGEMENTS}

The authors are supported by the National Nature Science Foundation of China (10833003), and thank CST for support.

\section{REFERENCES}

Dong, B., \& Han, J. L. 2013, PASA, 30, e032

Gan, H.-Q., \& Jin, C.-J. 2010, RAA, 10, 797

Nan, R. 2006, Sci. China G: Phy. \& Astron., 49, 129

Nan, R. et al. 2011, IJMPD, 20, 989

Qiu, Y. H. 1998, MNRAS, 301, 827 